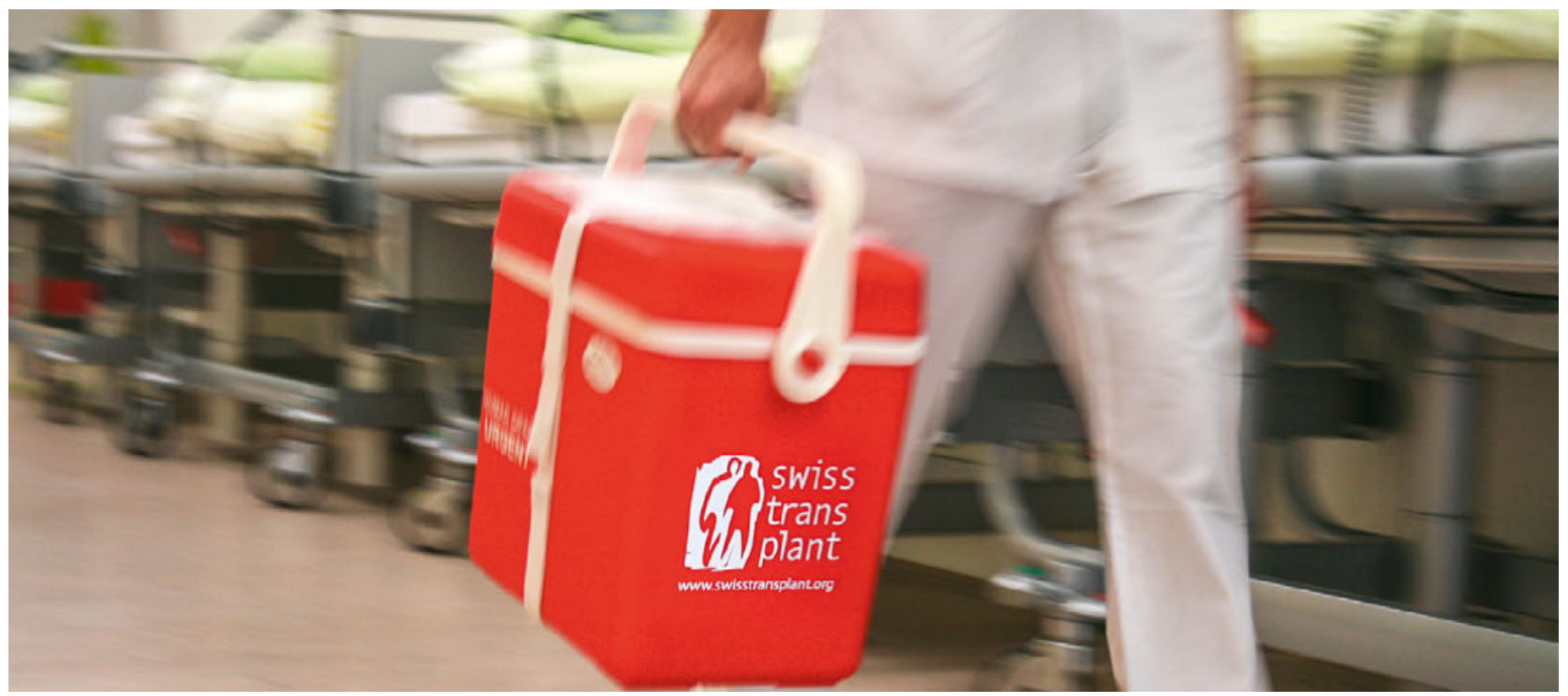

\title{
«Ein Register schafft Klarheit und Sicherheit»
}

\section{Matthias Scholer}

Wissenschaftsjournalist

Bei nur 10\% der Organspender liegt hierzulande eine schriftliche Willensäusserung vor. In allen anderen Fällen müssen die Angehörigen über eine Organentnahme entscheiden. Dies soll sich nun dank der Volksinitiative "Organspende fördern Leben retten» ändern. Geht es nach dem Willen der Initianten, soll in der Schweiz, analog zu den meisten europäischen Staaten, das Prinzip der vermuteten Zustimmung bei Organspenden implementiert werden.

\begin{abstract}
Ende 2013 lancierte der Bund einen Aktionsplan "Mehr Organe für Transplantationen». Können Sie eine erste Bilanz ziehen?

Ein wichtiges Ziel des Aktionsplans war es, das Thema Organspende auf Notfall- und Intensivstationen fest zu verankern. Die Zusammenarbeit mit den Spitälern wurde entsprechend intensiviert. Dank Schulungen des medizinischen Personals, der Vereinheitlichung von Abläufen und der Schaffung klar definierter Organisationsstrukturen in den Spitälern werden die Prozesse rund um Organspenden heute deutlich professioneller umgesetzt als noch vor wenigen Jahren.
\end{abstract}

\section{Schlägt sich der Aktionsplan auch in der Zahl spendenwilliger Personen nieder?}

Nein, leider nicht. Wir wollten auf das Niveau unserer Nachbarländer kommen. Wir peilten in der Schweiz 20 Organspender im primären Hirntod, sogenannte DBD oder deceased brain death donor, pro Million Einwohner an. Leider verharrt das Niveau auf tiefen 13 Organspendern pro Million. Und dies trotz verschiedenen flankierenden Massnahmen wie beispielsweise Plakataktionen und Fernsehwerbung. 
Wie erklären Sie sich die tiefe Bereitschaft, Organe zu spenden?

Die Gründe dafür sind sehr schwierig zu definieren. Grundsätzlich sind die Schweizer Organspenden gegenüber positiv eingestellt. Trotzdem sprechen sich 60\% der Angehörigen gegen eine Organfreigabe aus, wenn sie stellvertretend für einen Verstorbenen entscheiden müssen. Diese hohe Ablehnungsrate lässt sich unter anderem wohl darauf zurückführen, dass die Angehörigen den Wunsch des Verstorbenen meist nicht kennen und im Zweifelsfall von einer Freigabe der Organe absehen.

Im Oktober lancierte die Junior Chamber International eine Volksinitiative zur Förderung der Organspende. Ziel der Initiative ist es, in der Schweiz das Prinzip der vermuteten Zustimmung, welches in anderen Ländern als Widerspruchslösung bezeichnet wird, einzuführen. Wie ist die Unterschriftensammlung angelaufen? In der Startphase wurde die Initiative von der Bevölkerung gut aufgenommen. Nun folgt eine zweite Welle mit nationalen Sammeltagen. Die ersten Erfahrungen zeigen, dass es sich dabei um eine Initiative handelt, mit der sich sowohl die Gegner einer Organspende als auch Unentschlossene identifizieren können.

\section{Aber besteht nicht die Gefahr, dass ein Nicht-} zustande-Kommen der Initiative beziehungsweise eine Ablehnung an der Urne sich langfristig negativ auf die Spenderzahl niederschlägt?

Diese Frage haben wir uns anfänglich auch gestellt. Wir sind jedoch davon überzeugt, dass bereits die Unterschriftensammlung Diskussionen auf verschiedenen Ebenen auslöst und so Bevölkerungsschichten erreicht werden, die sich sonst kaum mit der Frage einer Organspende konfrontiert hätten. Die Tatsache, dass sich mehr Leute Gedanken zur Organspende machen und ihre persönlichen Ansichten mit ihren Angehörigen teilen, kann als Teilerfolg gewertet werden.

Im Jahr 2015 sprachen sich der Bund und der Ständerat gegen die vermutete Zustimmung aus. Sogar die Nationale Ethikkommission lehnt diese Regelung ab. Trotzdem wurde eine Volksinitiative lanciert. Eine Trotzreaktion?

Nein, das ist es sicherlich nicht. Auf politischer Ebene kam man dazumal zum Konsens, dass die Auswirkungen des bis Ende 2018 laufenden Aktionsplans abzuwarten sind. Wie schon erwähnt, konnten bis heute zwar die nötigen Strukturen und Prozesse auf Stufe Spital implementiert werden. Nun müssen wir über Massnahmen sprechen, dank denen sich die Spenderzahl steigern lässt. Dazu gehört auch ein Wechsel der
Modalitäten bei der Organspende. Dies ist schlussendlich ein gesellschaftlicher Entscheid, also soll die Bevölkerung auch mitsprechen.

Es werden immer wieder Stimmen laut, die eine Organentnahme bei Hirntoten als nicht vertretbar einstufen. Oder, dass eine Organentnahme den Sterbeprozess nachteilig beeinflusse. Können Sie diese Sorgen nachvollziehen?

Persönlich kann ich das Hirntodkonzept gut nachvollziehen. Ich bin mir aber bewusst, dass einzelne Kolleginnen und Kollegen das Konzept als solches hinterfragen. Genau deshalb soll auch jeder, der seine Organe nicht spenden will, diesen Wunsch ausdrücklich und unmissverständlich in einem Register hinterlegen können. Die herkömmliche Spenderkarte wird so modernisiert und vor allem auch besser zugänglich.

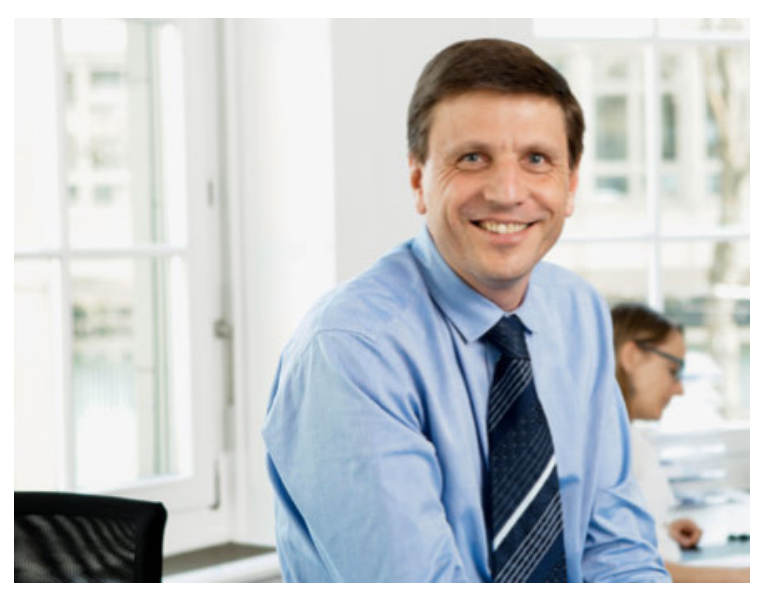

Franz Immer würde das Prinzip der vermuteten Zustimmung bei Organspenden begrüssen.

\section{Finden die meisten Organentnahmen} bei Hirntoten statt?

Wir gehen von 300 bis 350 Todesfällen auf Schweizer Intensivstationen aus, welche die Kriterien für eine Organspende im primären Hirntod erfüllen. Seit 2011 wurde die Organentnahme nach einem Herz-Kreislauf-Stillstand im Rahmen von Therapieabbrüchen auf Intensivstationen wieder eingeführt.

\section{Und wie ist in diesen Fällen die Organentnahme geregelt?}

Grundsätzlich handelt es sich dabei um Patienten, welche die Hirntodkriterien nicht erfüllen, aber im Rahmen eines Therapieabbruchs eine hohe Sterbewahrscheinlichkeit aufweisen - das heisst, bei welchen innert 120 Minuten nach Therapieabbruch ein HerzKreislauf-Stillstand eintritt. Bei diesen Patienten kann eine Organentnahme erfolgen, nachdem mittels Echo- 
kardiographie nachgewiesen wurde, dass das Herz mindestens 5 Minuten nach Herz-Kreislauf-Stillstand kein Blut mehr auswirft, gefolgt von einer Todesfeststellung im 4-Augen-Prinzip gemäss den Richtlinien der SAMW.

\section{Nach der Einführung der vermuteten Zustimmung gäbe es zwar ein entsprechendes Register. Aber viele Personen wollen sich zeitlebens gar nicht mit der Frage einer Organspende auseinandersetzen.}

Ich habe Mühe damit, dass man es den eigenen Angehörigen zumutet, sich stellvertretend zu einer Frage zu äussern, der man sich selbst nicht aussetzen möchte.

Eine Spende sollte doch eine altruistische Geste sein. Müsste man bei einer vermuteten Zustimmung nicht analog zum Impfzwang von einem Organspendezwang sprechen?

Der Systemwechsel bringt keinen Zwang, nur eine Verschiebung hin zur aktiven Selbstbestimmung. Jede Person soll für sich persönlich entscheiden, ob die eigenen Organe nach dem Tod anderen Mitmenschen zur Verfügung gestellt werden oder nicht. Ein Register schafft in diesem Punkt Klarheit und Sicherheit. Damit werden die Angehörigen massiv entlastet. In Frankreich kennt man das Prinzip der vermuteten $\mathrm{Zu}$ stimmung schon länger. Dort hat sich gerade mal ein Prozent der Bevölkerung explizit gegen eine Organentnahme ausgesprochen. Die Ablehnungsrate durch Angehörige liegt, wie übrigens auch in Italien und Österreich, bei tiefen $30 \%$.
Bedeutet dies, dass die Angehörigen bei einem fehlenden Negativeintrag im Register die Entscheidung tragen müssen?

Ja. In einem solchen Fall findet immer ein Gespräch mit den Angehörigen statt. Dabei geht es immer darum, den Wunsch des Verstorbenen zu evaluieren. Dieser soll massgebend sein. Ist den Angehörigen nicht bekannt, dass sich der Verstorbene zeitlebens in irgendeiner Form gegen die Organspende geäussert hat, so geht man bei der vermuteten Zustimmung von der Einwilligung aus.

Ein anderer Ansatz wäre, dass nur Organspender auch im Krankheitsfall Organe erhalten. Was halten Sie von diesem Vorschlag?

Wer gibt, soll auch priorisiert werden. Für mich persönlich ein vertretbares Vorgehen. Es lässt sich jedoch nicht mit unseren gesetzlichen Grundlagen vereinbaren. Das Parlament hat sich auch gegen diesen Lösungsansatz ausgesprochen, weil damit Bevölkerungsgruppen diskriminiert würden, die sich nie mit dem Thema Organspende beschäftigt haben. Es ist leider in den meisten Fällen so, dass man sich erst mit dem Thema Organspende auseinandersetzt, wenn man selber ein Organ braucht. Dann schütteln die meisten Betroffenen den Kopf, weil sie nicht verstehen, dass es so wenige Spender gibt.

Bildnachweis

(c) Swisstransplant 